Acta Hispanica (2020) Supplementum II: 933-942

\title{
LOS HERMANOS DE LA SALLE Y SU VINCULACIÓN CON LAS POLÍTICAS EDUCATIVAS COLOMBIANAS EN EL SIGLO XX
}

\author{
Carmen Elvira Semanate Navia
}

Universidad Pedagógica y Tecnológica de Colombia

\begin{abstract}
Resumen: La Congregación de los Hermanos de las Escuelas Cristianas, conocidos más como Hermanos de La Salle, han hecho grandes aportes a la educación en Colombia. Dicha Comunidad llegó a Colombia en el año 1891, época conocida en la historia de Colombia como el período de la Regeneración. Aquel período requería impulsar nuevas políticas educativas en concordancia con el sistema de gobierno que había iniciado hacia el año 1886 y buscaba con afán la implementación de políticas educativas acordes con la nueva constitución en donde se fomentaran nuevos valores educativos y tuviera como centro la religión católica. De esa manera los Hermanos de la Salle fundaron inicialmente algunas escuelas en la ciudad de Bogotá entre las que se conocen: la Escuela Apostólica, el Colegio de San Bernardo y el Noviciado de Chapinero. Hacia el año 1896 se funda el Instituto de La Salle en el barrio Egipto de la misma ciudad. La penetración del proyecto educativo lasallista obedeció al interés de aquella comunidad por fomentar enseñanzas prácticas que el estado colombiano requería en aquel momento. El sistema educativo de los Hermanos de La Salle buscó desarrollar una nueva educación moralizadora, concebida desde de una visión pragmática con fuerte énfasis confesional, es decir, desde la acción educadora de naturaleza católica, ejercida a partir de las enseñanzas modernas. Así mismo, es importante destacar el aporte que esta comunidad hizo en el Congreso pedagógico de 1917 donde se expusieron trabajos de investigación notables para el mejoramiento de la educación en Colombia.
\end{abstract}

Palabras clave: educación, misión lasallista, América Latina y el Caribe.

\begin{abstract}
The Congregation of the Brothers of the Christian Schools, better known as Hermanos de la Salle, have made great contributions to education in Colombia. This Community arrived in Colombia in 1891, a time known in the history of Colombia as the period of Regeneration. That period required the promotion of new educational policies in accordance with the system of government that had begun towards the year 1886 and eagerly sought the implementation of educational policies in accordance with the new constitution where new educational values were fostered and centered on the Catholic religion. Thus, the Brothers de la Salle initially founded some schools in the city of Bogotá, among which are known: the Apostolic School, the Colegio de San Bernardo and the Novitiate of Chapinero. By 1896 the De La Salle Institute was founded in the Egypt district of the same city. The penetration of the Lasallian Educational Project obeyed the interest of that community to promote practical teachings that the Colombian state required at that time. The educational system of the Brothers of La Salle sought to develop a new moralizing education, conceived from a pragmatic vision with a strong confessional emphasis, that is, from the educative action of a Catholic nature, exerted from modern teachings. Likewise, it is important to highlight the contribution that this community made in the pedagogical Congress of 1917 where notable research works were exhibited for the improvement of education in Colombia.
\end{abstract}

Keywords: Education, Lasallian Mission, Latin America and the Caribbean.

Acta Hispanica, Hungría, Supplementum II: 933-942, 2020, ISSN: 1416-7263 | 933 
Los Hermanos de La Salle y su vinculación con las políticas educativas colombianas en el siglo XX

\section{Introducción}

Es importante remontarnos un poco atrás para observar el origen de la Comunidad Religiosa denominada Hermanos de las Escuelas Cristianas, más conocidos como Hermanos de la Salle. Así mismo, observamos que, en Europa, a partir de Napoleón Bonaparte en el siglo XIX, se organizó la universidad como un monopolio del estado, con una intención puramente utilitaria y profesionalizante, según los ideales educativos y politécnicos que profesaba. Lo que resulta a partir de allí es un organismo al servicio estatal que la financia y organiza, y que fija no solo sus planes de estudio, su administración y el nombramiento de profesores, sino hasta el concepto de moral pública que ha de inculcar a sus discípulos. Dicho modelo fue imitado en América Latina, a raíz de la Independencia. La investigación dejó de ser un quehacer de la Universidad, y se reservó exclusivamente a las academias.

A finales del siglo XIX el asunto fue cambiando y en la década del sesenta del siglo $\mathrm{XX}$, aquel modelo hizo crisis, y se recupera para la Universidad su concepción unitaria, disciplinaria e investigativa. La universidad moderna surge del modelo que Guillermo de Humboldt obtuvo de las universidades alemanas, gracias al principio de la libertad académica. En la Universidad de Halle, donde se destaca el magisterio de Christian Wolff, se produce un extraordinario desarrollo en casi todas las ramas científicas a principios del siglo XIX, se produjo una aglomeración de grandes intelectuales alemanes: Schiller, Schelling, Fichte, Hegel y Humboldt. El modelo de la Universidad de Berlín es la antítesis del modelo napoleónico: el núcleo esencial de la universidad está constituido por la indisoluble unión de la ciencia objetiva y la formación subjetiva. Toda pretensión económica, social o estatal sobre la universidad debe ser rechazada. Lo único que cuenta es la investigación científica y la formación humana (Tünnermann Bernheim, 2000: 36).

Con Humboldt la universidad recupera el dominio de la ciencia y la investigación que había sido acaparado por las Academias de especial importancia en el desarrollo de la educación superior norteamericana fue la Ley Morrill de 1862 en tiempos del presidente Lincoln, mediante la cual el gobierno federal otorgaba tierras de su propiedad a los Estados que estuviesen dispuestos a crear colegios de agricultura y artes mecánicas. Estos colegios se transformaron después en las universidades estatales que se caracterizaron por su énfasis en las disciplinas agrícolas y tecnológicas y sus fuertes servicios de extensión. Pero el aporte más sobresaliente de la educación universitaria norteamericana a la organización de las universidades en el mundo entero fue la introducción del departamento como unidad académica básica, en sustitución de la cátedra aislada y unipersonal.

El Instituto Universitario de La Salle, nació con la edad moderna. Creció y se fortaleció en la búsqueda de una armonía entre la razón y la fe, dedicado a los hijos de los artesanos y de los pobres. En la práctica, el Instituto asume la responsabilidad de educar una nueva clase social que surge con el devenir de la modernidad, la clase media. La teoría y la praxis escolar tuvo que transformarse, y con todos los matices que se pueden 
hacer hoy sobre la interpretación que en la época tenían los términos artesanos y pobres, es absolutamente claro que el Instituto nunca entendió en estos términos a los hijos de los burgueses y de los ricos. La Revolución Francesa puso fin a un tipo de escuela dedicada a los artesanos y pobres y recuperó la experiencia educativa del Instituto para sus fines (Rodríguez Echeverría, 2009: 14-42).

\section{Instituto de La Salle en la época moderna}

Quiero resaltar que San Juan Bautista de La Salle no solo fue un personaje revolucionario de la pedagogía o un filántropo que se dedicó a educar a las familias más desfavorecidas del momento, se considera todo un protagonista que supo llegar a la mente y al corazón de las gentes, y transformó con su método educativo la cultura y mentalidad de los habitantes de su entorno en la época.

De esta manera, se reflexiona que la promoción humano-cristiana, llevada a cabo en el desarrollo sistemático e inteligente en la escuela de los Hermanos Cristianos tuvo injerencia en el hecho social y político del momento como fue la toma de la Bastilla 1789.

La restauración del Instituto en Francia, en la época de Napoleón, introdujo otro elemento importante en la vida de la Congregación, en la medida en que los Hermanos comenzaron a ser funcionarios de estado. Este régimen los llevó a ubicarse en los pensionados y en las instituciones de educación secundaria, de la cual no se ocuparon en el siglo anterior. La modalidad institucional adoptada fue la de colegio, la cual fue extendiéndose en todas partes; así, desde 1880 los Hermanos Cristianos pasaron a muchos países de América Latina, con el modelo de colegio que se fue propagando. Las escuelas gratuitas que constituyeron su participación primigenia fue en el siglo XVIII que luego acompañan un modelo mixto durante el siglo XIX (Rodríguez Echeverría, 2009: 14-42).

\section{Surgimiento de los Colegios de La Salle}

En la historia de la universidad, como lo afirma Ojeda 2002, se conoce que desde sus orígenes medievales contó con cuatro facultades: artes liberales, medicina, derecho y teología. La facultad de artes liberales era realmente una propedéutica y proporcionaba la cultura general indispensable para toda especialización. Correspondían sus estudios a lo que es hoy el bachillerato o secundaria preuniversitaria. Para llegar a la universidad, era menester pasar por estudios hechos en las llamadas escuelas de gramática, equivalentes a la enseñanza primaria de hoy, pero en todo caso debían demostrar que sabían leer, escribir y tener rudimentos del latín.

El ingreso a la universidad tenía lugar entre los trece y catorce años. Se sabe igualmente que los jóvenes estudiantes sintieron muy pronto la necesidad de agruparse, y como provenían de diversas regiones, los de un mismo origen alquilaban un local común y formaban una comunidad, en la que a menudo cenaban juntos con sus maestros. 
Los Hermanos de La Salle y su vinculación con las políticas educativas colombianas en el siglo XX

Personas caritativas tuvieron la idea de fundar establecimientos para albergar gratuitamente a los estudiantes pobres. Esta parece ser la orientación filosófica de los colegios Lasallistas, en los cuales se admitía a determinado número de becarios. Al principio, estos colegios fueron muy modestos y eran anexos de un hospital o de una casa religiosa, pero poco a poco se convirtieron en grandes fundaciones y transfirieron los dominios generales de la facultad de artes a la experiencia formativa que ofrecían a los estudiantes, y se convirtieron en internados (Ibarra Russi, 2009: 148).

Como lo afirma Ibarra, los Hermanos del Instituto de La Salle, incorporados por Napoleón a la dinámica educativa del imperio, articularon los colegios como experiencia preuniversitaria preparatoria de los estudiantes para la universidad, que en la dinámica napoleónica sufrió una transformación radical, al ser transformada en entidad de estado, como se ha dicho anteriormente. Este hecho permite inferir que los Hermanos de La Salle dieron un paso, en el siglo XIX, tan complejo y difícil respecto de la universidad como centro de educación superior, ya que la asumieron en su seno educativo y como parte sustantiva de sus modelos de formación. Si bien no tuvieron universidades, sí desarrollaron, por medio de los colegios, una comprensión directa de la enseñanza de la ciencia, la técnica, la ética y la estética y prepararon jóvenes para la formación universitaria en el ámbito de un pensamiento colegial.

Vale la pena señalar que los Hermanos sostenían una tradición pedagógica reconocida y sustentada al servicio de la enseñanza, por una parte, las escuelas llamadas de gramática que ellos transformaron a partir de su responsabilidad con la escuela gratuita y, por otra, la necesidad de perfeccionar el saber enseñar como elemento sustantivo, que los llevó a formular el método de enseñanza simultánea, la enseñanza en lengua materna en lugar del latín y formas pedagógicas prácticas que preparaban para la vida. Estos elementos son de alguna manera un contenido que se transforma mediante la práctica de los Hermanos como maestros y se condensa en una recuperación sistemática de mejores teorías y prácticas educativas, que conformaron la base conceptual de la transformación del oficio de maestro de profesión en maestro de guía de las escuelas (Ibarra Russi, 2009: 149).

El Instituto de los Hermanos de La Salle no solo desplegó un conocimiento en un ambiente pedagógico, sino que su misión fue más visionaria y crearon las escuelas normales como estructura de dominio para la formación de los maestros. Organizaron las escuelas en el casco urbano y en el rural, buscaron la manera de formar maestros que fueran capaces de vivir la interculturalidad. Esto permitió que el estado tuviera credibilidad en la formación que impartía este grupo de religiosos consagrados para el servicio de la educación.

\section{Llegada de los Hermanos Cristianos a Colombia}

Para 1880 la situación política de Colombia había empezado a alejarse de los excesos anticlericales de los liberales y a acercarse hacia una estructura de gobierno más conservadora. Fue el año en que en Colombia Rafael Núñez fue elegido presidente. Su 
elección representaba un cambio político regional importante con respecto a la base de poder de Tomás Cipriano de Mosquera, y un cambio ideológico que favorecía el tradicionalismo en la política y en la estructura social.

En 1884 Rafael Núñez fue reelegido presidente de la República, y como consecuencia del enfrentamiento entre los radicales y la coalición conservadora, se presentó una guerra civil en el año de 1885 (España, 1985). En ella resultaron derrotados los radicales, y entonces los miembros de la alianza convocaron un Consejo Nacional de Delegatarios para elaborar una nueva carta fundamental. En Colombia la Constitución de 1886 dispuso en su artículo 38 que "la Religión Católica, Apostólica, Romana, es la de la Nación; los poderes públicos la protegerán y harán que sea respetada como esencial elemento del orden social [...]". A su vez el artículo 40 estableció que "es permitido el ejercicio de todos los cultos que no sean contrarios a la moral cristiana ni a las leyes. Los actos contrarios a la moral cristiana o subversivos del orden público, que se ejecuten con ocasión o pretexto del ejercicio de un culto, quedan sometidos al derecho común" (Restrepo Piedrahita, 1991: 400). En materia educativa, el artículo 41 reguló que "la educación pública será organizada y dirigida en concordancia con la Religión Católica. La instrucción primaria costeada con fondos públicos será gratuita y no obligatoria" (España, 1985).

Como se puede observar en el movimiento de Regeneración de Núñez, los conservadores y la Iglesia se convirtieron en la plataforma política. Así, la nueva constitución, de carácter centralista, inició su vigencia en el año 1886. Esta constitución le devolvió el poder al ejecutivo central y se restauró el prestigio y el poder de la Iglesia católica. Colombia que se convirtió en una nación con gran fuerza conservadora, católica y centralizada. Coyuntura que llevó a firmar un concordato con la Santa Sede en 1887 (Mejía Arango, 2007). Este concordato definió la relación de Colombia con la Iglesia católica y aseguró que Colombia fuera una nación católica, realidad que habría de influenciar la cultura, la educación y la política hasta 1930.

En estas circunstancias, los Hermanos de las Escuelas Cristianas más conocidos como Hermanos de La Salle, fueron invitados al país por Monseñor Bernardo Herrera Restrepo Obispo de Medellín para dirigir un colegio de varones en la capital antioqueña, fue de esa manera como la comunidad religiosa ingresó a Colombia en el año de 1890. Contexto del movimiento regenerador, que buscaba retomar la participación del pensamiento católico en la organización de la sociedad.

Lo anterior fue muy significativo y así el gobierno colombiano le ofreció a los Hermanos Cristianos la dirección de la Escuela Normal de Bogotá. Los religiosos profesaban la tradición educativa confesional francesa originada en San Juan Bautista de La Salle. Cabe anotar que entre las congregaciones masculinas que llegaron en aquel momento a la República de Colombia, fue la Congregación de los Hermanos de las Escuelas Cristianas como la más importante; su llegada coincidió con la reforma escolar católica. Los Hermanos de La Salle garantizaban una enseñanza conforme a los lineamientos del Vaticano. Los religiosos se hicieron famosos por dirigir las escuelas 
Los Hermanos de La Salle y su vinculación con las políticas educativas colombianas en el siglo XX

normales y los colegios técnicos (Helg, 2001: 78) debido a esta experiencia que ellos demostraron fue por lo que el gobierno les ofreció la dirección de la Escuela Normal Central de Institutores y del Instituto Técnico Central de Bogotá que administraron libremente hasta 1930, lo cual significaba una responsabilidad muy grande pero que con mucha dedicación la comunidad religiosa supo responder a las necesidades y requerimientos del país.

\section{Florecimiento de la educación lasallista en Colombia}

En la enseñanza secundaria clásica, los Hermanos de La Salle, asumieron la educación de las élites y fue ahí donde la Congregación progresó. Fundó colegios privados en diferentes ciudades del país con una salvedad que en cada colegio funcionaba una escuelita de catecismo para niños pobres. Los Hermanos de la Salle alcanzaron tanto prestigio en pedagogía que se colocaron en primer puesto y quedaron a nivel de los colegios de los Jesuitas.

Los lasallistas trajeron métodos pedagógicos novedosos avanzados. Además, la Congregación aprovechó para educar a jóvenes e ilustrarlos en la fe para que continuaran su formación en los noviciados que ya habían fundado en Bogotá, Medellín y Barranquilla, aspecto que les favoreció dado que iban reemplazando a los religiosos franceses. El legado Lasallista no se traduce solo en una formación bajo los preceptos católicos, Apostólicos y Romanos, sino que en ellos se conjuga el humanismo y las ciencias experimentales como una fusión en la formación integral de sus educandos (López López, 1991).

Al estallar la guerra de los Mil Días, se desorganizaron las facultades universitarias y gran número de establecimientos educativos fueron convertidos en cuarteles, pero una vez pasada la triste guerra civil, el director de instrucción pública José Joaquín Casas, insistió en la propuesta que desde 1892 el gobierno le había formulado a los religiosos de la Salle para que se encargaran de la dirección de la Escuela Normal de Cundinamarca.

Por lo tanto, el 14 de marzo de 1902 se firmó el contrato entre el presidente José Manuel Marroquín y el Hno. Gabriel María, Superior General del Instituto de los Hermanos Cristianos para que enviara a Colombia cinco hermanos que se encargarían de asumir la dirección de la Normal, así como de redactar su reglamento que estaría de acuerdo con el criterio y el espíritu que animó al superior de La Salle, cuando fundó el Seminario de maestros en 1685 - primera Normal del Instituto-, en una casa separada de la comunidad donde los maestros vestidos de negro de acuerdo con su reglamento aprendían a cantar gregoriano, a leer y escribir a la perfección. 


\section{La Escuela Normal Central de Institutores de Bogotá Bajo la responsabilidad de los Hermanos de La Salle}

Durante la administración del general Rafael Reyes, el 5 de febrero de 1905, cuando inició labores La Escuela Normal Central de Institutores con treinta alumnos maestros becados por la nación y el departamento de Cundinamarca. Sin embargo, y debido a los progresos pedagógicos obtenidos por los Hermanos de La Salle, llegaron estudiantes de todo el país hasta Bogotá a pesar de existir en cada capital de departamento una Escuela Normal de carácter oficial.

Como medio de divulgación la Normal publicó la Revista Pedagógica que editó desde 1918 hasta 1927 dando a conocer los adelantos pedagógicos, modelos de enseñanza, prácticas y orientaciones para la catequesis, la educación física, la estética, etc. De la brillante nómina de religiosos que fueron maestros en la Escuela Normal merecen desatarse los hermanos Justo Ramón, Gastón María, Luis Gonzaga, Estanislao Luis, Benildo Matías y Bladelin León, quienes por su cultura hicieron de este centro la base para que la comunidad Lasallista continuara impartiendo estudios superiores. Así mismo, se destacaron los alumnos José Eustasio Rivera y Eduardo Carranza, orientados hacia las letras por el hermano francés Juan Teodoro en la Escuela que alcanzó a impartir instrucción pedagógica durante 30 años.

La formación de maestros fue una responsabilidad muy grande y ante todo dieron una respuesta a tan importante petición del gobierno, puesto que se estaba atendiendo al clamor popular por una comunidad religiosa como eran los Hermanos de la Salle quienes consiguieron con destino al asilo de San José, maquinaria y materias primas para los talleres donde los niños desamparados debieron trabajar y estudiar bajo la dirección del Hermano llamado Lediberien. Pronto el asilo fue convertido en Escuela Central de Artes y Oficios y la Educación fue reglamentada por el General Rafael Reyes, el 9 de febrero de 1905 compuesta de dos secciones un curso preparatorio que duraba dos años y los cursos de artes y oficios, tres años.

Los progresos técnicos conseguidos por los Hermanos de La Salle fueron evaluados por las comisiones de Inspección Educativa, lo que favoreció la expedición del decreto No 2006 del 24 de noviembre de 1916, firmado por el presidente José Vicente Concha, el cual permitió a la Escuela de Artes y oficios otorgar los títulos de ingeniero en electricidad y artes mecánicas; ingeniero en electricidad e industrias textiles; e ingeniero en electricidad y arte industrial decorativa (López López, 1991).

Con el anterior reconocimiento quedaron establecidos los estudios superiores dentro de la enseñanza católica Lasallista; el arte decorativo fue cambiado por arquitectura, adquiriendo esta escuela gran prestigio nacional, especialmente por el talento artístico de su director el Hermano Geric Benjamin, egresado de la Escuela de Arquitectura de san Lucas (Bélgica) y de quien el pintor Luis Alberto Acuña, dijo que "me complace reconocer como mi primer maestro y guía inicial en mi carrera de escultor. Pues escultor fue él esencialmente, bien que no en forma exclusiva ya que también canceló 
Los Hermanos de La Salle y su vinculación con las políticas educativas colombianas en el siglo XX

certeramente los metales, como primoroso tecnicismo de orfebre, y sobre todo, proyectó y dirigió construcciones con cabal conciencia de arquitecto", tal como lo señala López López (1991).

Por otra parte es importante resaltar que el presidente Marco Fidel Suárez le cambió el nombre a la Escuela de Artes y Oficios por el Instituto Técnico Central según el decreto 721 de 4 de abril de 1919, nombre que conservó con sus programas superiores hasta el año de 1931 cuando fue unido a la Facultad de Ingeniería Civil en medio de una gran polémica, ya que los actos del gobierno liberal se veían a través del prisma de la revancha para el conservatismo que no se resignaba a dejar el poder después de 45 años de hegemonía.

\section{Los Hermanos Cristianos y el Congreso Pedagógico Colombiano de 1917}

Fue en el Congreso Pedagógico del año 1917 donde los trabajos emanados de la experiencia educativa en los centros educativos fueron presentados como muestra fehaciente de la tarea pedagógica de los Hermanos de La Salle. Allí, los Hermanos comenzaron a socializar algunos conocimientos referentes a sus investigaciones y experiencias pedagógicas, demostrando con ello, la dinámica cognoscitiva subyacente a sus prácticas. Esto permite visualizar de entrada que el componente pedagógico no permanecía inerte en un trayecto histórico determinado, por el contrario, obedecía a múltiples implicaciones y factores que tuvieron su origen en períodos históricos anteriores (Florencio-Andrés, 1965: 15).

Desde aquel momento se buscó coordinar la enseñanza que se impartía, desde la infantil hasta la industrial, profesional y artística para que simultáneamente se enseñara agricultura, industria y comercio por considerarlas las tres fuentes de la riqueza pública y privada. El autor Antonio José Uribe al referirse a las universidades pidió que su educación fuese más completa; urgió la necesidad de fomentar la formación de seminarios o centros de estudio entre los alumnos, de unir al profesorado para que se evaluaran de acuerdo con el progreso de las facultades y de estrechar los vínculos entre las universidades de la República, más en sentido patriótico que académico, por el recuerdo de la separación de Panamá.

La marcada orientación Lasallista se deduce por el nuevo espíritu práctico que identificaba sus planes de estudio. Pero, hablando del Congreso Pedagógico Nacional del año 1917 en número de 30 religiosos de esta comunidad asistieron al citado Congreso Pedagógico. con marcada orientación francesa. Por eso fueron las propuestas del Hno. Agustín sobre la enseñanza profesional técnica y sus aplicaciones en Colombia, lo mismo que la enseñanza comercial del Hno. Idimael Henry.

El aporte de los Hermanos Lasallistas que mostraron en el Congreso Pedagógico nacional del año 1917 fue notable y se puede concretar en varios aspectos. Sin embargo, se hace especial mención en referentes tales como: el espíritu práctico que promulgaron en sus ponencias, que presentaron en el Congreso, en donde mostraban que la Educación 
debía contener metodologías aplicadas en desarrollo de la diversidad de temas. Se hizo énfasis sobre la enseñanza profesional y técnica que formaran en los jóvenes el saber hacer. Además, se mostró allí la importancia de una enseñanza comercial que orientara a la población en saberes prácticos que los formara en nuevos saberes para el trabajo y en áreas que ayudaran al desarrollo del país. Por otra parte, la Revista Pedagógica de la Normal Central de Institutores, Apuntes y Consejos Pedagógicos, Boletines de Colegios, además de textos escolares, y algunos elementos pedagógicos necesarios en las instituciones educativas.

Así mismo, con insistencia en sus ponencias mostraron la necesidad de aplicar nuevas metodologías en la enseñanza con el fin de crear una nueva pedagogía que fortaleciera las instituciones educativas y les diera a los estudiantes herramientas para sus aprendizajes y pudieran concluir sus enseñanzas en tareas que los habilitaran en el campo laboral y en técnicas de trabajo.

\section{Conclusiones}

Los Hermanos de las Escuelas Cristianas llegaron a Colombia a finales del siglo XIX gracias a la invitación de monseñor Bernardo Restrepo y a los diferentes acuerdos entre la Iglesia Católica y el Gobierno. Desde su llegada y a lo largo de la primera mitad del siglo XX los Lasallistas llevaron a cabo diferentes fundaciones escolares en todos los sectores de la educación y así se responsabilizaron de los niveles de: primaria, secundaria y profesional, tanto pública como privada. Además, estuvieron presentes en la formación de técnicos, maestros, políticos, empresarios industriales y religiosos colombianos. Por consiguiente, esta fue una comunidad religiosa que se destacó en el campo educativo colombiano.

Podríamos afirmar que el Instituto de La Salle ha sido el centro de aquella filosofía y ha buscado el desarrollo educativo de la República y ha enfrentado las crisis de la educación que conducen a la consolidación de un sistema educativo integrado entre la educación primaria, secundaria y superior. De igual manera los aportes de creación de los métodos simultáneos de educación, formación de maestros, enseñanza en lenguas propias y educación centrada en la persona y en la vida, abrieron camino a la modernización de las universidades. Desde la perspectiva de ciclos propedéuticos, la educación técnica y tecnológica constituyó elemento fundamental en la educación superior que marcó un derrotero significativo para el país.

\section{Referencias bibliográficas}

España, Gonzalo (1985). La Guerra Civil de 1885. Núnezy la derrota del radicalismo. Bogotá: El Ancora Editores.

Florencio, Rafael - Andrés, Bernardo (1965). Los Hermanos de La Salle en Colombia. Bodas de Diamante 1890-1965. Medellín: Bedout. 
Los Hermanos de La Salle y su vinculación con las políticas educativas colombianas en el siglo XX

Helg, Aline (2001). La educación en Colombia: 1918-1957. Bogotá: Plaza \& Janes.

Ibarra Russi, Oscar Armando (2009). Aportes del lasallismo al desarrollo de la educación superior en la modernidad latinoamericana. Revista de la Universidad de La Salle, 49. 143 156.

López López, Héctor (1991). Historia de la Universidad de la Salle. Bogotá: Ediciones Unisalle. Mejía Arango, Lázaro (2007). Los radicales. Bogotá: Universidad Externado de Colombia.

Restrepo Piedrahita, Carlos (1991). Constituciones políticas nacionales de Colombia. Bogotá: Universidad Externado de Colombia.

Rodríguez Echeverría, Fsc. Hermano Álvaro (2009). La misión lasallista a nivel terciario. Revista de la Universidad de La Salle, 49. 14-42.

Rodríguez Echeverría Fsc Hermano Álvaro (2006). La educación universitaria dentro de la Misión Lasallista. Revista Universidad de La Salle, 42. 10-16.

Tünnermann Bernheim, Carlos (2000). Universidad y Sociedad. Balance histórico y perspectivas desde Latinoamérica. Caracas: Universidad Central de Venezuela. 